\title{
CircCRIM1 promotes ovarian cancer progression by working as ceRNAs of CRIM1 and targeting miR-383-5p/ZEB2 axis
}

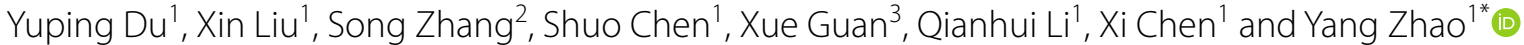

\begin{abstract}
Background: Ovarian cancer is the leading cause of death in patients with gynecologic cancer, and circular RNAs (circRNAs) are involved in cancer progression. However, there are limited studies on the roles of circRNAs in ovarian cancer.

Methods: We designed divergent and convergent primers, used sanger sequencing and RNase $R$ digestion to verify the source of circCRIM1. We detected the expression of circCRIM1 and its parental gene cysteine rich transmembrane BMP regulator 1 (CRIM1) in ovarian cancer and normal ovarian samples via qRT-PCR. MTT viability assay, apoptosis assay, wound healing assay and invasion assay were used to investigate the function of circCRIM1 and CRIM1 in ovarian cancer cell lines OVCAR3 and CAOV3. Mice xenografts experiment was performed. Bioinformatics predicted the microRNAs that bond with circCRIM1 and CRIM1, and dual luciferase reporter system confirmed it. Rescue experiments of microRNAs mimics transfection on the basis of circCRIM1 over-expression were carried out to uncover the mechanism by which circCRIM1 played cancer-promoting roles in ovarian cancer.
\end{abstract}

Results: CircCRIM1 was derived from CRIM1 by back-splicing. CircCRIM1 and CRIM1 had higher expression in ovarian cancer than in normal ovarian tissues, and both of them promoted ovarian cancer progression in vitro. In vivo circCRIM1 promoted the growth of tumors. CirCCRIM1 and CRIM1 had a positive correlation relationship in the same cohort of ovarian cancer tissues. Bioinformatics predicted and dual luciferase assay confirmed circCRIM1 and CRIM1 bond with miR-145-5p, and circCRIM1 bond with miR-383-5p additionally. CircCRIM1 positively affected the expression of CRIM1. After circCRIM1 was over-expressed, miR-145-5p mimics transfection reversed the expression of CRIM1. Western blot discovered circCRIM1 positively affected the expression of zinc finger E-box binding homeobox 2 (ZEB2). Rescue experiments found miR-383-5p mimics reversed ZEB2 expression and the cancer-promoting effects of circCRIM1.

Conclusions: CircCRIM1 bond with miR-145-5p to work as competing endogenous RNA (ceRNA) of CRIM1, and circCRIM1 bond with miR-383-5p to improve the expression of ZEB2 in ovarian cancer. CircCRIM1 and CRIM1 promoted the ovarian cancer progression and supplied a novel insight into the researches of ovarian cancer.

Keywords: circCRIM1, CRIM1, Ovarian cancer, miR-145-5p, miR-383-5p

*Correspondence: yida.zhaoyang@163.com

1 Department of Obstetrics and Gynecology, Department of Gynecologic Oncology Research Office, Key Laboratory for Major Obstetric Diseases of Guangdong Province, The Third Affiliated Hospital of Guangzhou Medical University, Guangzhou 510150, China

Full list of author information is available at the end of the article

\section{Background}

Ovarian cancer is the leading cause of death in patients with gynecologic cancer. Owing to the lack of an early diagnostic strategy, this seriously declines survival. Especially in serous ovarian cancer, majority of patients are diagnosed at stage III (51\%) or IV (29\%) [1-3]. Therefore, original author(s) and the source, provide a link to the Creative Commons licence, and indicate if changes were made. The images or other third party material in this article are included in the article's Creative Commons licence, unless indicated otherwise in a credit line to the material. If material is not included in the article's Creative Commons licence and your intended use is not permitted by statutory regulation or exceeds the permitted use, you will need to obtain permission directly from the copyright holder. To view a copy of this licence, visit http://creativecommons.org/licenses/by/4.0/. The Creative Commons Public Domain Dedication waiver (http://creativeco mmons.org/publicdomain/zero/1.0/) applies to the data made available in this article, unless otherwise stated in a credit line to the data. 
it is extremely important to seek early markers of ovarian cancer.

Circular RNAs (CircRNAs) working as a novel class of non-coding RNAs, played critical roles during the process of initiation and development of cancers. They consisted of a circular configuration through typical $5^{\prime}$ to $3^{\prime}$ - phosphodiester bond. Lacking free terminus, thus they gained much more stable existence in the cells compared to linear RNAs [4]. To date, the researches on the roles of circRNAs have been continuously extended and deepened, which was involved in multiple diseases, including cancers $[5,6]$, cardiovascular diseases $[7,8]$, diabetes [9], autoimmune diseases and so on [10]. CircRNAs exerted their function through multiple ways. They could regulate the expression of their parental genes by transcriptional and post-transcriptional pathways, act as microRNAs "sponges", bind with RNA-binding proteins to form RNA-protein complexes and even have open reading frames (ORFs) to be translated into proteins/ peptides [11-16]. According to the previous researches, many circRNAs have identified their important roles in the cancers [17-19].

CircRNAs were mainly derived from precursor mRNAs by the way of back-splicing [20-23]. Hsa_circ_0002346 (referred to as circCRIM1) was derived from exon2-4 of cysteine rich transmembrane BMP regulator 1 (CRIM1) that was a bone morphogenetic protein family antagonist $[24,25]$. In the existing studies, CRIM1 had a higher expression in gastric cancer and drug-resistant myeloid leukemia cells [26, 27]. Furthemore, CRIM1 promoted adhesion and migration of lung cancer cells and promoted invasion of prostate cancer cells [28, 29]. However, there was no comparative report about the function of CRIM1 and circCRIM1 in ovarian cancer. Therefore, this paper focused on the role and mechanism of circCRIM1 and its parental gene coding protein CRIM1 in ovarian cancer.

\section{Methods}

\section{Specimens collection}

Normal ovarian $(n=24)$ and ovarian cancer tissues $(n=130)$ were collected from surgical excision in the First Affiliated Hospital of China Medical University (Shenyang, Liaoning, China), and patients had no previous radiotherapy or chemotherapy. This project was approved by the ethic committee of China Medical University (No:2018-132) and written consents were obtained from all patients or their families.

\section{Cell lines and transfection}

The human ovarian cancer cell lines OVCAR3 and CAOV3 were cultured in RPMI 1640 (HyClone, Logan, UT, USA) added with 10\% FBS and 1\% penicillin/ streptomycin. All siRNAs, plasmids and microRNA mimics transfection used Lipofectamine 3000 reagent (Invitrogen, Carlsbad, USA). The sequences of siRNA of CRIM1 (Sigma-Aldrich, St. Louis, MO, USA): sense CUC AGUACUCCCUCCAUUUdTdT, anti-sense AAAUGG AGGGAGUACUGAGdTdT. The detail of plasmids was shown in the Tables S5, S6, S7, S8.

\section{RNase $\mathbf{R}$}

Total RNA was extracted from OVCAR3, then $5 \mu \mathrm{g}$ of total RNA was formulated into a $20 \mu \mathrm{l}$ system with RNase $\mathrm{R}$ (Epicentre, Madison, USA) for RNase R (+) group and RNase-Free water for RNase R (-) group, and incubated at $37^{\circ} \mathrm{C}$ for $0,10,20,30 \mathrm{~min}$. The reaction was terminated at $70^{\circ} \mathrm{C}$ for $10 \mathrm{~min}$. The $5 \mu \mathrm{l}$ of each product was reverse transcribed and amplified following qRT-PCR procedures.

\section{qRT-PCR}

Total RNA was extracted from tissues or cells using RNA isolater Total RNA Extraction Reagent (Vazyme Biotech, Nanjing, China) and then was reverse transcribed to cDNA with reverse transcription kit (Promega, Madison, Wisconsin, USA). cDNA was amplified using 2xSYBR Green qPCR Master Mix (Bimake, Houston, TX, USA). $18 \mathrm{~s}$ or $\beta$-actin was utilized to normalize the expression. The detail of primer sequences was shown in the Tables S9.

\section{MTT viability assay}

Cells were seeded in 96-well plates at a density of 3000 cells/well. Cells were transfected after overnight, if it needed. And then each well added MTT $(5 \mathrm{mg} / \mathrm{ml}) 20 \mu \mathrm{l}$ at indicated time periods. After further incubation of $4 \mathrm{~h}$, culture medium was removed and $150 \mu \mathrm{l}$ DMSO was added. The absorbance was obtained at $490 \mathrm{~nm}$ using a microplate spectrophotometer (BioTek Instruments, Winooski, VT).

\section{Apoptosis assay}

Cells were collected and washed with PBS. $100 \mu \mathrm{l}$ of $1 \times$ buffer, $5 \mu$ l of Annexin V-FITC and $5 \mu \mathrm{l}$ of PI (BD Biosciences, San Jose, CA, USA) were used in the dark for transient transfected cells. And for stable transfected cells, 7AAD and annexin V-PE (BD Biosciences) were used.

\section{Wound healing assay}

Cells were seeded in 6-well plate. And after overnight scratched it with a $200 \mu \mathrm{l}$ tip. Cells were cultured in $5 \%$ serum medium after washed away floating cells with PBS. Cells were transfected if it needed. Cells migration was observed and photographed at $0 \mathrm{~h}$ and $48 \mathrm{~h}$. The wound 
healing rate was measured by the way as follows: (area at $0 \mathrm{~h}-$ area of wound at $48 \mathrm{~h}$ )/ area at $0 \mathrm{~h}$.

\section{Invasion assay}

Cells diluted by serum-free medium were seeded in the top compartment of the Transwell cell culture chambers (BD Bioscience) at a density of $4 \times 10^{4}$ cells per well, and precoated membrane with matrigel (1:10) before inoculation. Complete medium $600 \mu \mathrm{l}$ was layered in the bottom compartment. Cells were transfected if it needed. After being incubated $48 \mathrm{~h}$, the cells were fixed and dyeing.

\section{Xenografts assay}

All 4-week-old female BALB/c nude mice (Vital River Laboratories, Beijing, China) were randomly divided into two groups $(n=6)$ and were fed under the standard condition. The vector and plasmid transfected cells were injected subcutaneously at a concentration of $1 \times 10^{7}$ cells/ $200 \mu$ l. The tumor volumes were monitored and mice were sacrificed when the maximum tumor diameter was close to $2 \mathrm{~cm}$. Tumor volumes were calculated using the formula: (length $\times$ width $^{2}$ ) / 2. All animal experiments conformed to the National Institutes of Health Guide for the Care and Use of Laboratory Animals and were supported by the China Medical University Animal Care and Use Committee.

\section{Western blotting}

Cells were lysed by RIPA buffer and the concentration was determined. Proteins were run on sodium dodecyl sulphate-polyacrylamide gels relying on molecular weight and then were wet transferred to Hybond membranes. 5\% fat-free milk was used to block nonspecific combination at room temperature for $2 \mathrm{~h}$, followed by incubation at $4{ }^{\circ} \mathrm{C}$ overnight with primary antibodies against CRIM1(Cat No. bs-21654R, Biosis, China), Flag (Cat No. F3165, Sigma-Aldrich), ZEB2(Cat No. 140261-AP, Proteintech, China) and $\beta$-actin (Cat No. 205361-AP, Proteintech). TBST washed membranes 4 times and were incubated with the secondary antibody for $2 \mathrm{~h}$ at room temperature. An enhanced chemiluminescence (Santa Cruz Biotechnology, Santa Cruz, CA, USA) was used to visualize the bands.

\section{Dual luciferase assay}

The sequences of wild type circCRIM1 and microRNA binding sites mutant circCRIM1 were inserted into dual luciferase reporter plasmid psicheck2.0 (HANBIO, Shanghai, China). Similarly, the wild type sequences of CRIM1 mRNA 3'UTR and mutant sequences were inserted into vector GV272 (GENE, Shanghai, China). MicroRNA mimics or miR-NC was co-transfected with above wild and mutant plasmids into HEK293 cells. After 48 h, Dual-Luciferase Reporter System (Promega) was used to measure luciferase activities.

\section{Statistical analyses}

All data were expressed as mean \pm standard deviation. At least three independent experiments were performed. Two groups were compared with the two-sided student's $t$ test. Correlation was calculated using Pearson's correlation analysis.
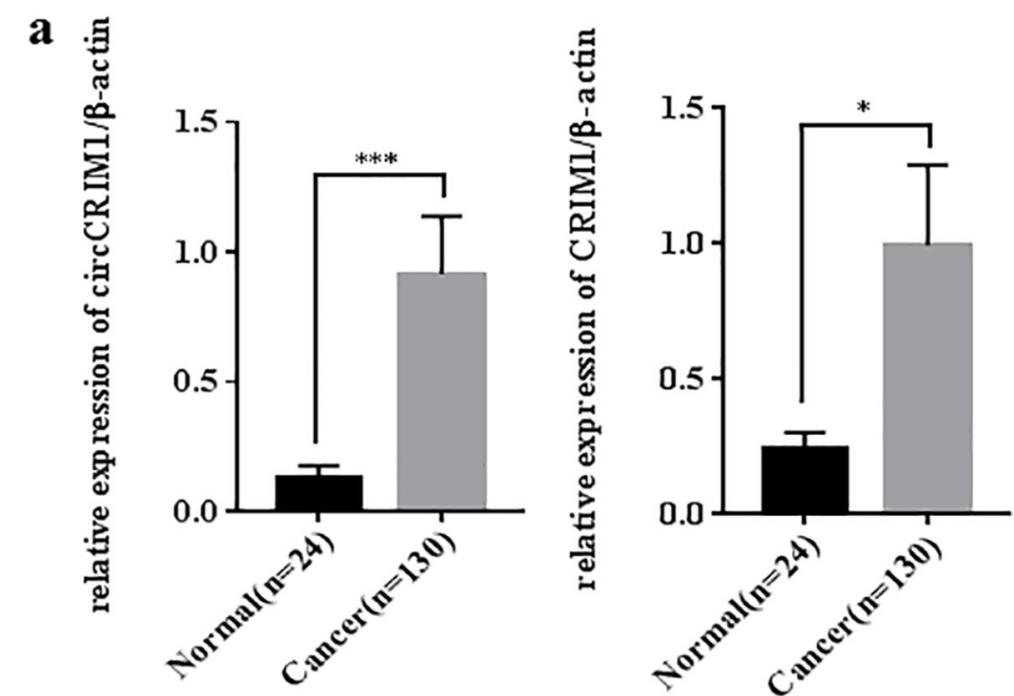

Fig. 1 CircCRIM1 and CRIM1 are up-regulated in ovarian cancer. Both circCRIM1 and CRIM1 were up-regulated in ovarian cancer (a). Data are shown as the mean $\pm \mathrm{SD}$. ${ }^{*} P<0.05,{ }^{* *} P<0.01$, ${ }^{* *} P<0.001$ and ${ }^{* * *} P<0.0001$ 


\section{Results}

\section{CircCRIM1 is derived from CRIM1 by back-splicing}

CircCRIM1 with a $538 \mathrm{nt}$ circular structure was predicted by CircRNA Db to be derived from CRIM1 exon2-4 (Fig. S1a). The back-splicing junction was amplified with divergent primers and verified by Sanger sequencing (Fig. S1b). CircCRIM1 was more resistant to RNase $\mathrm{R}$ than CRIM1 and $18 \mathrm{~s}$ (Fig. S1c; $p<0.05$ ), thus confirmed its ring structure. We designed 2 pairs of convergent primers and one pair of divergent primers to amplify CRIM1 and circCRIM1 from cDNA and gDNA and verified
circCRIM1 was only derived from RNA but not DNA (Fig. S1d). These results suggested that circCRIM1 was derived from CRIM1 by back-splicing.

\section{Both circCRIM1 and CRIM1 are up-regulated in ovarian cancer}

The expression of circCRIM1 was significantly up-regulated in ovarian cancer tissues compared with normal ovarian tissues. Furthemore, we detected CRIM1 expression in the same cohort of ovarian cancer tissues and normal ovarian tissues, the expression of CRIM1 mRNA

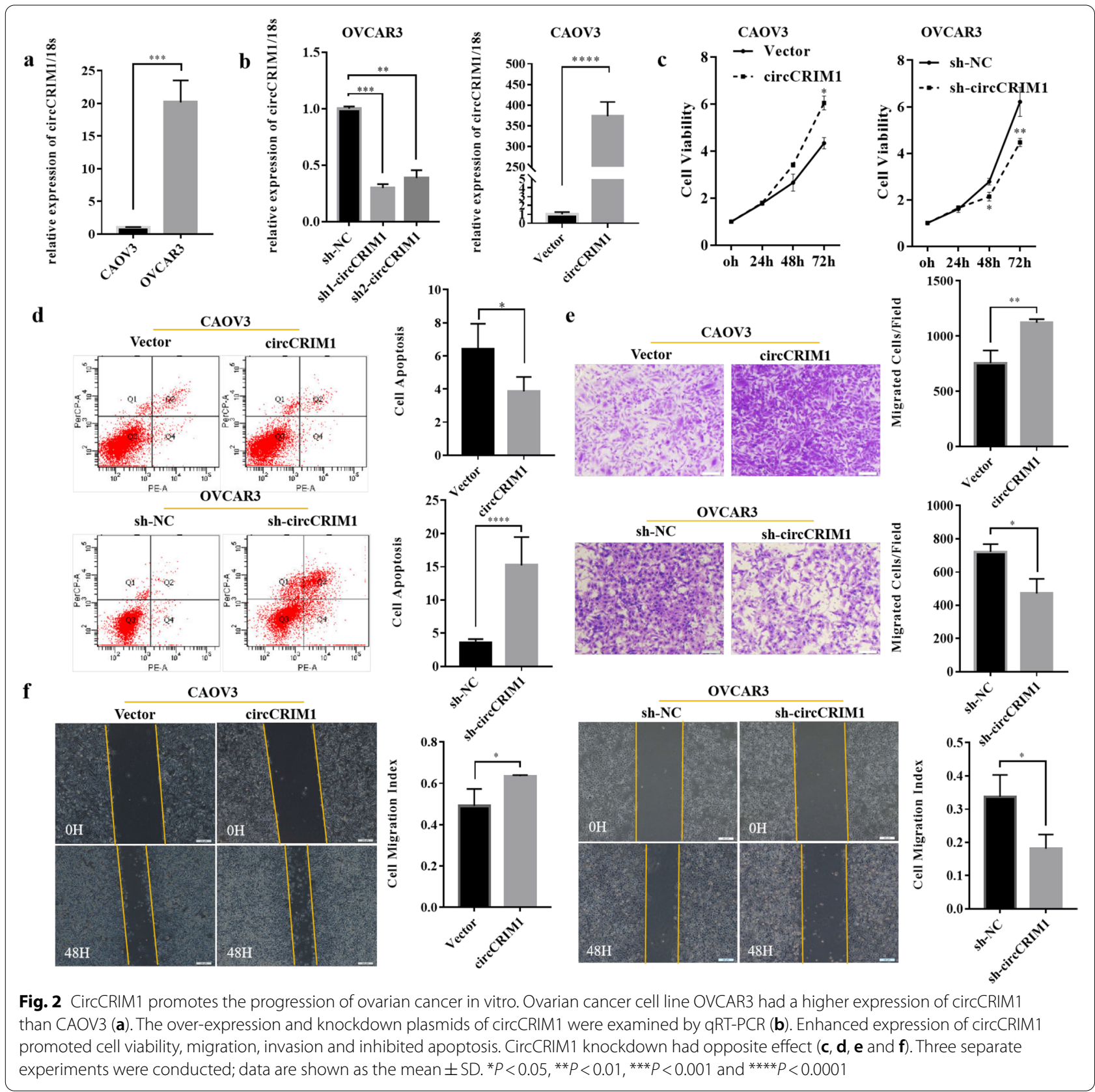


was higher in ovarian cancer tissues than that in normal ovarian tissues (Fig. 1a; $p<0.05$ ). The detail of expression information was shown in the Tables S1, S2, S3, S4.

\section{CircCRIM1 promotes the progression of ovarian cancer in vitro}

To evaluate the functional role of circCRIM1 in ovarian cancer, circCRIM1 over-expression and knockdown plasmids were respectively constructed. Ovarian cancer cell line OVCAR3 had a higher expression of circCRIM1 than ovarian cancer cell line CAOV3 (Fig. $2 \mathrm{a} ; P<0.05$ ). The knockdown plasmid sh1 had stronger suppression effect and we used it for the following experiments. The overexpression plasmid was transfected into CAOV3 and verified with qRT-PCR (Fig. 2b; $P<0.05$ ). Enhanced expression of circCRIM1 promoted cell viability, migration, invasion and inhibited apoptosis. On the other hand, circCRIM1 knockdown inhibited cell viability, migration, invasion and promoted apoptosis (Fig. 2c, d, e and f; $P<0.05$ ).

\section{CircCRIM1 promotes the progression of ovarian cancer in vivo}

In order to investigate the role of circCRIM1 in tumor growth in vivo, the CAOV3 cells transfected with vector or circCRIM1 over-expression plasmid were respectively subcutaneously injected into the right flanks of mice. The tumor volumes of circCRIM1 over-expression group were larger than that of control group (Fig. 3a and b; $P<0.05)$. The result revealed that circCRIM1 promoted the tumor growth in vivo.

\section{CRIM1 promotes the progression of ovarian cancer}

Through design of siRNA, CRIM1 was knocked down in ovarian cancer cell lines CAOV3 and OVCAR3. Interference efficiency of CRIM1 siRNA was verified with qRT-PCR and western blot (Fig. 4a and b; $P<0.05$ ). After CRIM1 was interfered, the viability, migration, invasion ability of ovarian cancer cell lines decreased and apoptic rate increased (Fig. 4c, d, e and f; $P<0.05$ ).

\section{CircCRIM1 positively regulates the expression of CRIM1 through miR-145-5p}

We analyzed the expression of CRIM1 mRNA and circCRIM1 in the same cohort of ovarian cancer tissues, and found that there was a positive correlation relationship between circCRIM1 and CRIM1 (Fig. 5a; $P<0.05$ ). We also found that CRIM1 expression was positively influenced by circCRIM1 (Fig. 5b). TargetScanHuman predicted that miR-145-5p was likely to combine with 3'UTR of CRIM1 and Circular RNA Interactome predicted miR145-5p had complementary sequences targeting circCRIM1 (Fig. 5c). Dual luciferase reporter assay showed that the co-transfection of miR-145-5p mimics and 3'UTR fragment of CRIM1 inhibited luciferase activity compared to miR-NC and 3'UTR of CRIM1 co-transfection, while miR-145-5p binding site mutation abolished this effect. Similarly, miR-145-5p mimics and wild type circCRIM1 reporter plasmid co-transfection inhibited the luciferase activity compared to miR-NC and wild type circCRIM1 co-transfection, while miR-145-5p binding site mutation abolished this effect (Fig. $5 \mathrm{~d} ; P<0.05$ ).

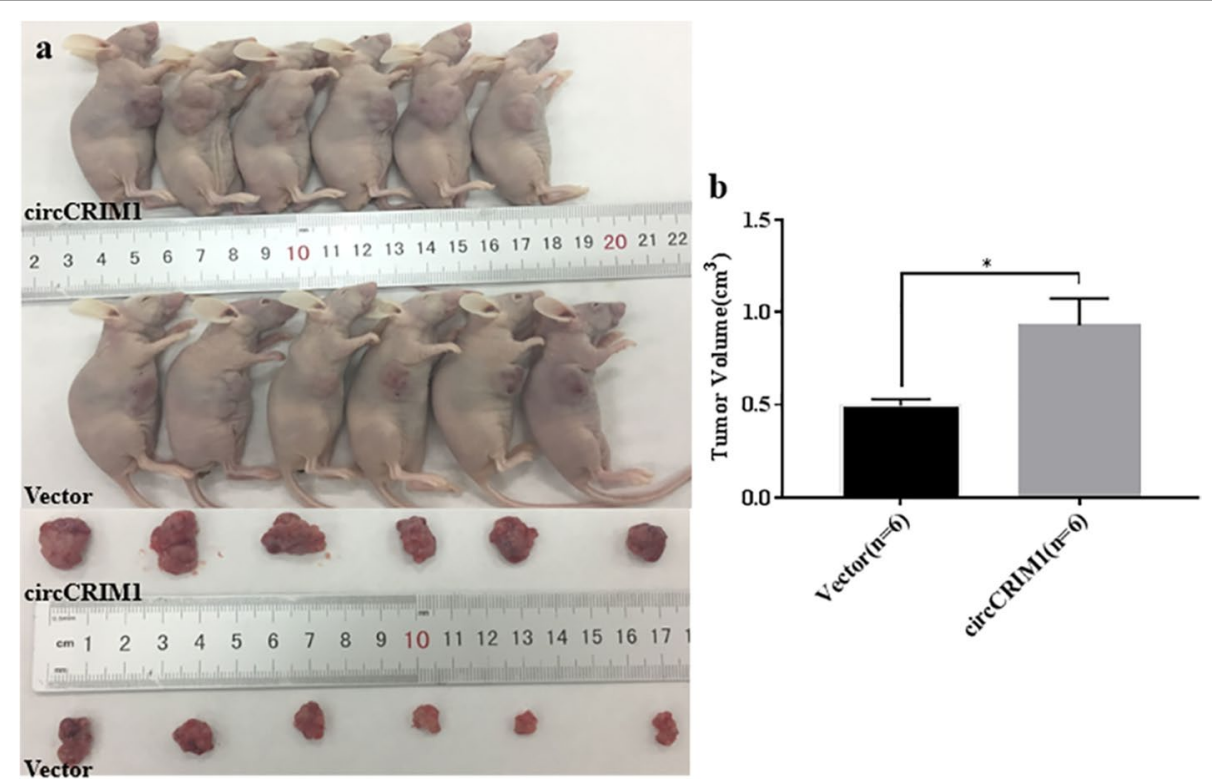

Fig. 3 CircCRIM1 promotes the growth of ovarian cancer in vivo. The group that upregulated circCRIM1 had more bulky tumor volume compared to vector group (a and $\mathbf{b}$ ). Data are shown as the mean \pm SD. ${ }^{*} P<0.05,{ }^{* *} P<0.01,{ }^{* *} P<0.001$ and ${ }^{* * *} P<0.0001$ 


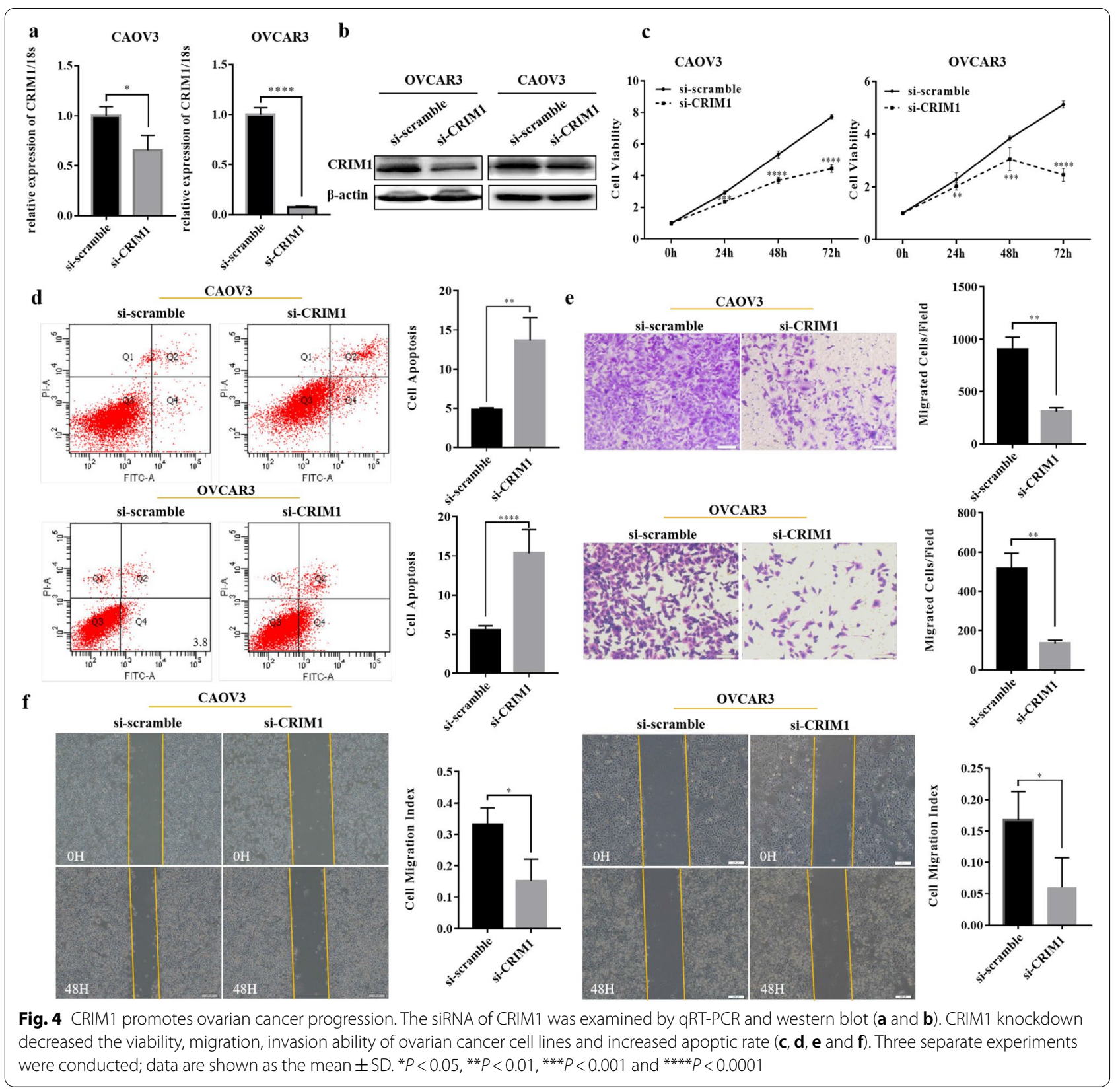

Furthermore, miR-145-5p mimics treatment reversed the expression of CRIM1 after over-expression of circCRM1, and miR-145-5p mimics over-expression ability was verified (Fig. 5e and f; $p<0.05$ ). These results indicated that the expression of CRIM1 was positively regulated by circCRIM1 through miR-145-5p.

\section{CircCRIM1 may encode 188aa protein}

CircRNA Db predicted that circCRIM1 had a putative ORF with size 188aa (Fig. 6a). To explore that whether circCRIM1 could encode protein, $3 \times$ flag sequences were added into putative ORF in original circCRIM1 over-expression plasmid and avoided frame shifting, stop codon disruption, junction disruption and IRES disruption (Fig. 6b). We used flag antibody to detect the expression of $188 \mathrm{aa}-3 \times$ flag fusion protein with predicted molecular weight around $27 \mathrm{kd}$. There was detectable protein band (Fig. 6c). We were trying to pull down 188 aa $-3 \times$ flag for mass spectrometry verification by means of immunoprecipitation with flag antibody. However, when the positive control was pulled down, 188 aa- $3 \times$ flag was unable to be pulled down (Fig. $6 \mathrm{~d}$ ). 

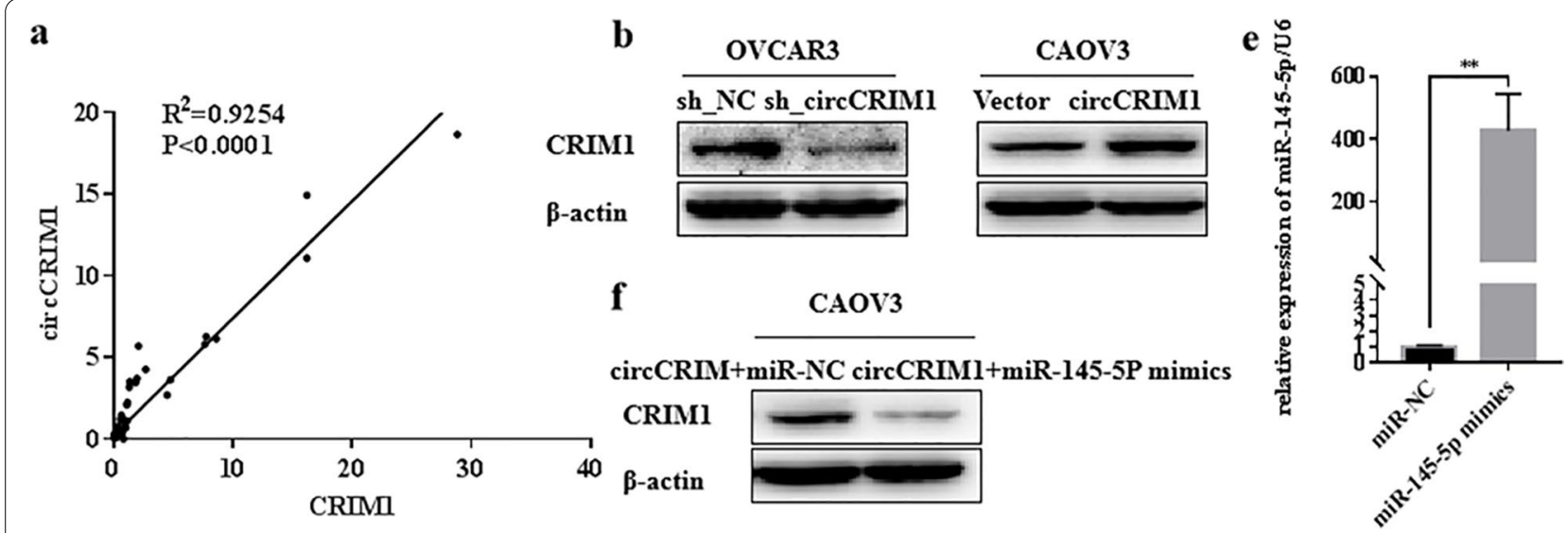

c Position 1574-1580 5'...UGGUUUGAAACAACUACUGGAAU...
of CRIMI 3' UTR

hsa-miR-145-5p 3' UCCCUAAGGACCCUUUUGACCUG

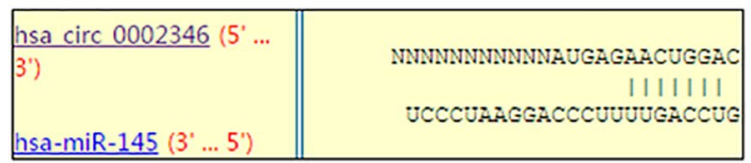

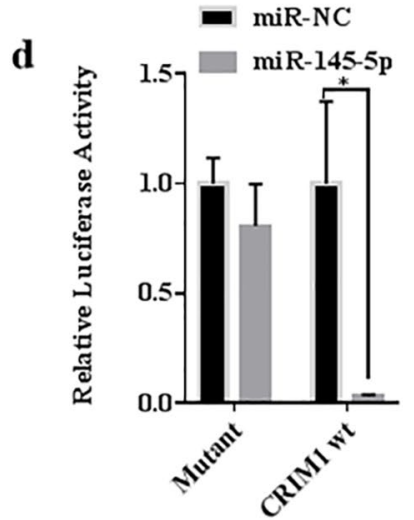

Fig. 5 CircCRIM1 positively regulates the expression of CRIM1 through miR-145-5p. QRT-PCR showed that there was a positive correlation relationship between circCRIM1 and CRIM1 (a). CRIM1 expression was obviously positively influenced by circCRIM1 (b). TargetScanHuman predicted miR-145-5p might combine with $3^{\prime} U$ TRs of CRIM1 and Circular RNA Interactome predicted miR-145-5p might bind with circCRIM1(c). Dual luciferase reporter assay verified it (d). MiR-145-5p treatment reversed the expression of CRIM1 after the over-expression of circCRM1(f). MiR-145-5p mimics efficiency was verified $(\mathbf{e})$. Three separate experiments were conducted; data are shown as the mean $\pm \mathrm{SD}$. ${ }^{*} P<0.05,{ }^{* *} P<0.01,{ }^{* * *} P<0.001$ and ${ }^{* * * *} P<0.0001$

In spite of this, $188 \mathrm{aa}$ and $3 \times$ flag sequences were introduced into protein expression vector and transfected into OVCAR3 (Fig. 6e). We found over-expression of 188aaflag promoted cell proliferation, invasion and migration in OVCAR3 (Fig. 6f, g and h; $P<0.05$ ).

\section{CircCRIM1 binds with miR-383-5p to promote ovarian cancer progression}

By using Circular RNA Interactome, we identified that miR-383-5p had complementary sequences targeting to circCRIM1 (Fig. 7a). The dual luciferase reporter assay was performed and found that miR-383-5p mimics and wild type circCRIM1 reporter plasmid co-transfection inhibited luciferase activity compared to miR-NC and wild type circCRIM1 co-transfection, while miR-383-5p binding site mutation abolished this effect (Fig. 7b;
$P<0.05)$. MiR-383-5p mimics over-expression ability was verified (Fig. 7c; $P<0.05$ ). MiR-383-5p has been reported to bind with ZEB2. Western blot found circCRIM1 enhancement or knockdown positively regulated ZEB2 (Fig. 7d). MiR-383-5p mimics treatment in CAOV3 that over-expressed circCRIM1 reversed the promotion effect of circCRIM1 on viability, migration, invasion ability and ZEB2 expression, increased apoptic rate (Fig. 7e, f, g, h and i; $P<0.05)$.

\section{Discussion}

By the way of cDNA and gDNA PCR, Sanger sequencing and RNase $\mathrm{R}$ digestion, we confirmed circCRIM1 was derived from exon2-4 of protein coding gene CRIM1 pre-mRNA by back-splicing. In our study, we identified that circCRIM1 was up-regulated in ovarian cancer compared with normal ovarian tissues. In order to 


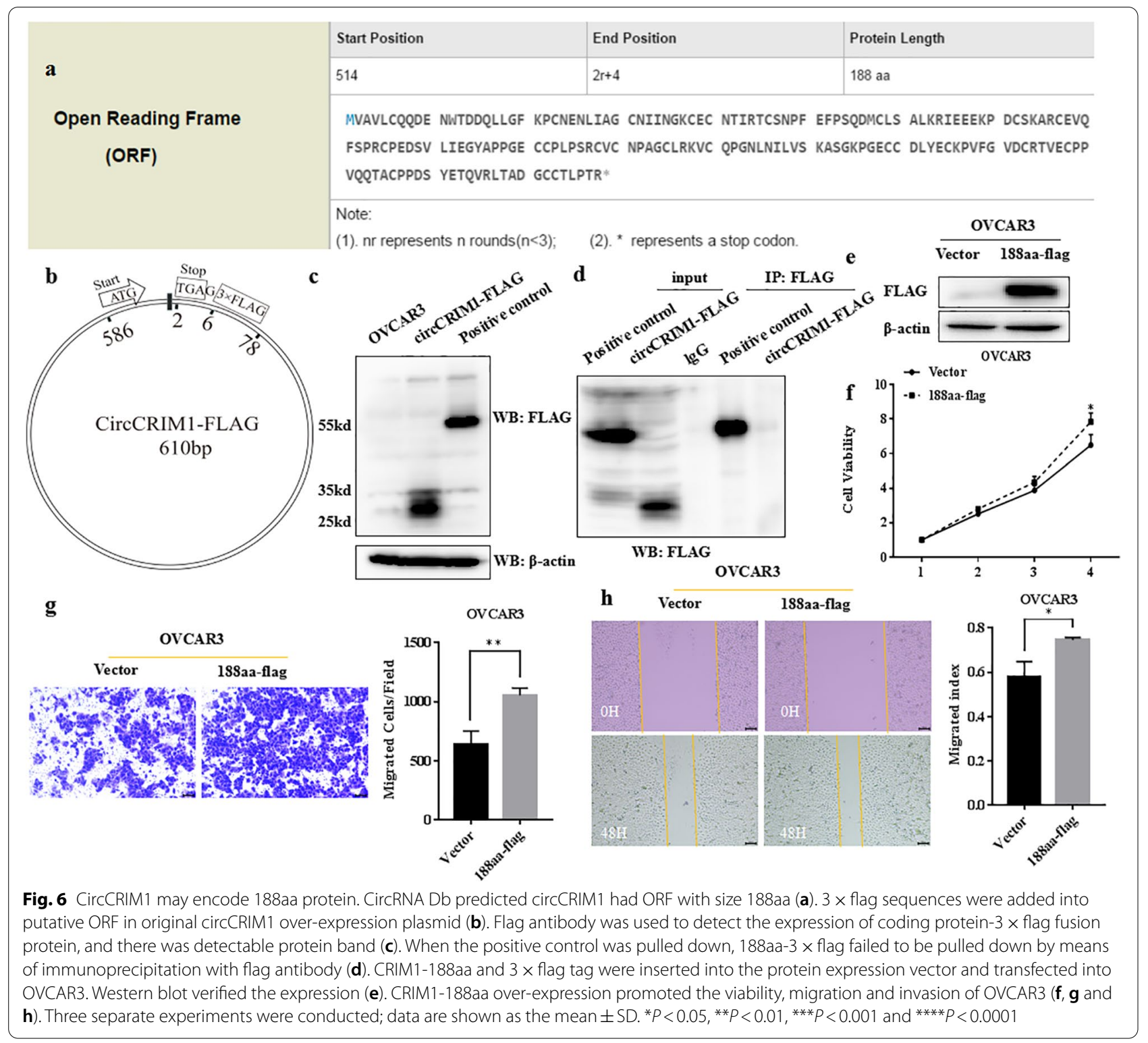

determine the function of circCRIM1 in ovarian cancer, we detected the expression levels of circCRIM1 in ovarian cancer cell lines CAOV3 and OVCAR3. We found that the circCRIM1 was higher in OVCAR3 than that in CAOV3. Thus, we chose CAOV3 to over-express and OVCAR3 to knock down the expression of circCRIM1. Functional experiments were carried out to explore the roles of circCRIM1 in ovarian cancer. We observed that circCRIM1 enhancement promoted cell viability, migration, invasion and inhibited apoptosis and circCRIM1 knockdown produced opposite effects. The nude mice xenograft assay was performed and suggested that circCRIM1 promoted the growth of tumors in vivo. All in all, circCRIM1 promoted the progression of ovarian cancer in vitro and in vivo.
A few articles have revealed the cancer-promoting effects of CRIM1, but there was no comparative study on the effects of CRIM1 in ovarian cancer. We detected the CRIM1 mRNA in the same samples of ovarian cancer and normal ovarian tissues as above. The results revealed that CRIM1 mRNA was higher in ovarian cancer tissues compared to normal ovarian tissues. Then we identified that CRIM1 interference inhibited cell viability, migration, invasion and promoted apoptosis in ovarian cancer cell lines. Thus, CRIM1 and circCRIM1 both played cancer-promoting roles in ovarian cancer.

As mentioned above, circRNAs could regulate the expression of their parental genes. We analyzed the expression levels of circCRIM1 and CRIM1 in ovarian cancer samples and found there was a positive 


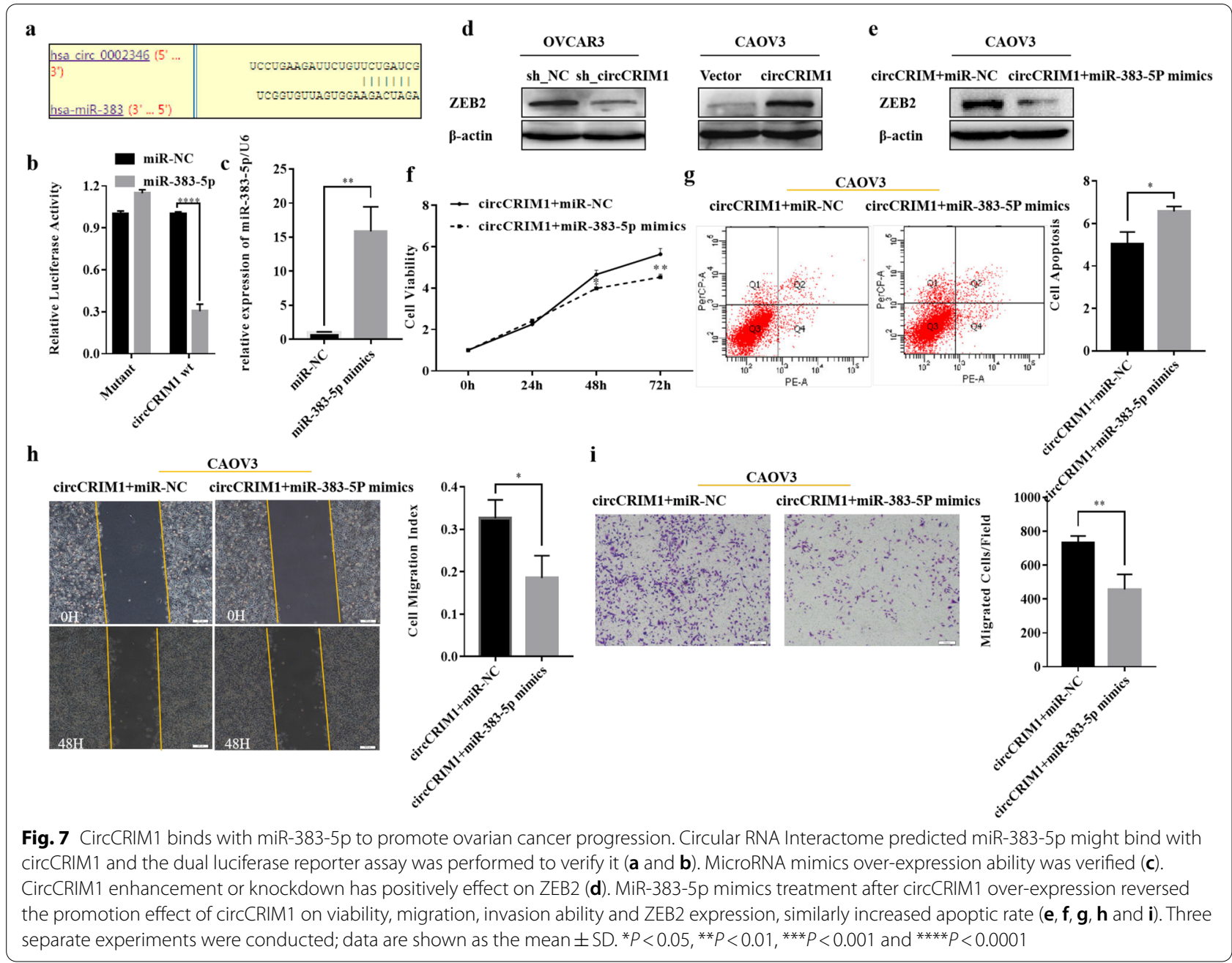

correlated relationship between circCRIM1 and CRIM1 mRNA. Apart from this, the over-expression and knockdown of circCRIM1 in ovarian cancer cell lines positively influenced CRIM1 expression. So how did circCRIM1 regulate the expression of CRIM1? We intersected microRNAs predicted by TargetScanHuman to bind to CRIM1 mRNA 3'UTR and microRNAs predicted by Circular RNA Interactome to bind to circCRIM1. It was found that miR-145-5p might bind to both CRIM1 mRNA 3'UTR and circCRIM1 [30-33]. Previous studies have found that the expression of miR$145-5 p$ in normal ovarian tissues is greater than in epithelial ovarian cancer, and up-regulated expression of miR-145-5p significantly inhibited proliferation, invasion and in vivo tumor formation, and induced apoptosis in ovarian cancer cell lines [34, 35]. And then the dual luciferase assay was performed to verify the combination of miR-145-5p and CRIM1 mRNA 3'UTR or circCRIM1. Furthermore, miR-145-5p mimics treatment decreased the expression of CRIM1 in the cells that over-expressed circCRIM1. Thus, it indicated that circCRIM1 worked as ceRNA of CRIM1 mRNA trough miR-145-5p.

Recent studies have proposed that some circRNAs containing ORFs could be efficiently translated into proteins or peptides. Circ-AKT3 encoded a new 174aa protein, which negatively regulated the PI3K / AKT pathway [36]. Circ-FBXW7 encoded a 185aa protein that reduced the half-life of c-Myc [37]. We sought to elucidate that whether circCRIM1 had the ability of encoding protein or peptide. CircRNA Db predicted that circCRIM1 with ORF started from 514nt had the possibility to produce a protein with length 188aa [38]. We added $3 \times$ flag sequences into putative ORF in original circCRIM1 over-expression plasmid and used flag antibody to hybrid with predicted 188aa-flag fusion protein which had a molecular weight about $27 \mathrm{kd}$. There was detectable protein band. Then we tried to pull down $188 \mathrm{aa}-3 \times$ flag for mass spectrometry verification by means of immunoprecipitation with flag antibody to confirm the existence 
of 188aa. However, when the positive control was pulled down, 188 aa- $3 \times$ flag failed to be pulled down. Its not clear if this was due to factors that the flag sequence was included in the 188aa sequence, the stability of the protein was poor, or something else. It required further exploration. In spite of this, 188 aa and $3 \times$ flag tag were inserted into the protein expression vector and transfected into OVCAR3. The over-expression of 188aa promoted cell viability, migration and invasion.

CircRNA usually exerted its function through the way of "sponge". Bioinformatic analyses indicated that circCRIM1 might target with miR-383-5p. By means of the dual luciferase assay, we confirmed that miR-383-5p combined with circCRIM1. Previous study found that miR-383-5p worked as tumor suppressor gene to suppress proliferation of ovarian cancer cells and inhibit progression of gastric cancer [39-41]. While ZEB2 worked as oncogenic genes in ovarian cancer to drive EMT transition, tumorigenesis and peritoneal metastasis $[42,43]$. The study found that ZEB2 expression in ascites of patients with high-grade serous ovarian cancer was higher than that of primary ovarian cancers. ZEB2 positively regulated the proportion of CD133+ cancer stem-like cells in epithelial ovarian cancer cells and promote the tumorigenesis and peritoneal metastasis capacity of CD133+ cancer stem-like cells. Bioinformatics, luciferase reporter system and western blot verified ZEB2 mRNA 3'UTR was the target of miR-383-5p [44]. Western blot was performed and we found the expression of ZEB2 was consistent with circCRIM1. Then rescue experiments were carried out. We found that miR-383-5p mimics transfection after circCRIM1 over-expression reversed the promotion of circCRIM1 on cell viability, migration, invasion and ZEB2 expression, and promoted apoptosis. Taken together, circCRIM1 bond with miR383-5p to up-regulate ZEB2 in ovarian cancer and played the cancer-promoting roles. In addition, in the following work, we could focus on the effect of circCRIM1 on ovarian cancer peritoneal metastasis.

In a word, circCRIM1 worked as ceRNA of CRIM1 through miR-145-5p, and bond with miR-383-5p to improve ZEB2 to play the cancer-promoting roles in ovarian cancer.

\section{Conclusion}

This article supplied a significant perspective on the roles of circCRIM1 in ovarian cancer. It bond with miR-383-5p to improve the expression of ZEB2 in ovarian cancer. Moreover, CRIM1, the parental gene of circCRIM1, promoted the progression of ovarian cancer. CircCRIM1 bond with miR-145-5p to work as ceRNA of CRIM1, and both of them offered a novel insight into researches of ovarian cancer.

\section{Abbreviations}

ceRNA: Competing endogenous RNA; circRNA: Circular RNA; CRIM1: Cysteine rich transmembrane BMP regulator 1; DMSO: Dimethyl sulfoxide; ORFs: Open reading frames; ZEB2: Zinc finger E-box binding homeobox 2.

\section{Supplementary Information}

The online version contains supplementary material available at https://doi. org/10.1186/s12958-021-00857-3.

Additional file 1 Supplemental Table 1. circCRIM1 expression in normal ovarian and ovarian cancer tissues. The detail information of circCRIM1 expression in normal ovarian and ovarian cancer tissues. Supplemental Table 2. CRIM1 expression in normal ovarian and ovarian cancer tissues. The detail information of CRIM1 expression in normal ovarian and ovarian cancer tissues. Supplemental Table 3. Correlation of circCRIM1 expression with different clinicopathological features of ovarian cancer. The detail information of the correlation of circCRIM1 expression with different clinicopathological features of ovarian cancer. Supplemental Table 4. Correlation of CRIM1 expression with different clinicopathological features of ovarian cancer. The detail information of the correlation of CRIM1 expression with different clinicopathological features of ovarian cancer. Supplemental Table 5. circCRIM1 expression plasmid construction. The information of circCRIM1 expression plasmid. Supplemental Table 6. The sequences of shRNA targeting circCRIM1. The information of circCRIM1 shRNA. Supplemental Table 7. circCRIM1-FLAG expression plasmid construction. The information of circCRIM1-FLAG expression plasmid. Supplemental Table 8. 188aa-flag expression plasmid construction. The information of 188aa-flag expression plasmid. Supplemental Table 9. The detail of primer sequences. The sequences of circ primer, convergent primer1, convergent primer2 and mRNA primer. Supplemental Figure 1. CircCRIM1 is derived from CRIM1 by back-splicing. CircCRIM1 was generated from exon2-4 of CRIM1 (a). Sanger sequencing verified the back-splicing junction of circCRIM1 (b). CircCRIM1 was more resistant to RNase R than CRIM1 and 18s (c). CircCRIM1 was only derived from RNA but not DNA (d)

\section{Acknowledgements}

Not applicable.

\section{Authors' contributions}

Yang Zhao, Yuping Du and Xin Liu conceived the study. Yuping Du wrote the manuscript. Yuping Du, Xin Liu, Song Zhang, Shuo Chen, Xue Guan, Qianhui $\mathrm{Li}$ and Xi Chen performed experiments and statistics. All authors read and approved the final manuscript.

\section{Funding}

This study was supported by National Natural Science Foundation of China (Nos. 81872115; 81772776), The Young Science-technology Talents Support Project of Guangzhou (No. X20200301068) and The Project for Key Medicine Discipline Construction of Guangzhou Municipality (No. 2021-2023-17).

\section{Availability of data and materials}

The datasets used and/or analysed during the current study are available from the corresponding author on reasonable request.

\section{Declarations}

\section{Ethics approval and consent to participate}

This project was approved by the ethic committee of China Medical University (No:2018-132) and obtained informed consents from patients or their families. All animal experiments conformed to the National Institutes of Health Guide for the Care and Use of Laboratory Animals and were supported by the China Medical University Animal Care and Use Committee.

\section{Consent for publication}

Not applicable. 


\section{Competing interests}

The authors declare that they have no competing interests.

\section{Author details}

${ }^{1}$ Department of Obstetrics and Gynecology, Department of Gynecologic Oncology Research Office, Key Laboratory for Major Obstetric Diseases of Guangdong Province, The Third Affiliated Hospital of Guangzhou Medical University, Guangzhou 510150, China. ${ }^{2}$ Department of Thoracic Surgery, The First Affiliated Hospital of China Medical University, Department of Environmental and Occupational Health, School of Public Health, China Medical University, Shenyang 110001, China. ${ }^{3}$ Department of Gynecology, The First Affiliated Hospital of China Medical University, Shenyang 110001, China.

Received: 8 August 2021 Accepted: 10 November 2021 Published online: 30 November 2021

\section{References}

1. Torre LA, Trabert B, DeSantis CE, Miller KD, Samimi G, Runowicz CD, et al Ovarian cancer statistics, 2018. CA Cancer J Clin. 2018;68:284-96.

2. Bowtell DD, Böhm S, Ahmed AA, Aspuria PJ, Bast RC Jr, Beral V, et al. Rethinking ovarian cancer II: reducing mortality from high-grade serous ovarian cancer. Nat Rev Cancer. 2015:15:668-79.

3. Morris CR, Sands MT, Smith LH. Ovarian cancer: predictors of early-stage diagnosis. Cancer Causes Control. 2010;21:1203-11.

4. Hsiao KY, Sun HS, Tsai SJ. Circular RNA - new member of noncoding RNA with novel functions. Exp Biol Med (Maywood). 2017;242:1136-41.

5. Zong Z-H, Du Y-P, Guan X, Chen S, Zhao Y. CircWHSC1 promotes ovarian cancer progression by regulating MUC1 and hTERT through sponging miR-145 and miR-1182. J Exp Clin Cancer Res. 2019:38:437.

6. Liu J, Liu H, Zeng Q, Xu P, Liu M, Yang N. Circular RNA circ-MAT2B facilitates glycolysis and growth of gastric cancer through regulating the miR-515-5p/HIF-1a axis. Cancer Cell Int. 2020;20:171.

7. Li H, Xu JD, Fang XH, Zhu JN, Yang J, Pan R, et al. Circular RNA circRNA_000203 aggravates cardiac hypertrophy via suppressing miR26b-5p and miR-140-3p binding to Gata4. Cardiovasc Res. 2020;116:1323-34.

8. Zhou LY, Zhai M, Huang Y, Xu S, An T, Wang YH, et al. The circular RNA ACR attenuates myocardial ischemia/reperfusion injury by suppressing autophagy via modulation of the Pink1/ FAM65B pathway. Cell Death Differ. 2019;26:1299-315.

9. Zhao Z, Li X, Jian D, Hao P, Rao L, Li M. Hsa_circ_0054633 in peripheral blood can be used as a diagnostic biomarker of pre-diabetes and type 2 diabetes mellitus. Acta Diabetol. 2017;54:237-45.

10. Li LJ, Zhu ZW, Zhao W, Tao SS, Li BZ, Xu SZ, et al. Circular RNA expression profile and potential function of hsa_circ_0045272 in systemic lupuserythematosus. Immunology. 2018;155:137-49.

11. Zhang Y, Zhang XO, Chen T, Xiang JF, Yin QF, Xing YH, et al. Circular intronic long noncoding RNAs. Mol Cell. 2013;51:792-806.

12. Ichinose J, Watanabe K, Sano A, Nagase T, Nakajima J, Fukayama M et al. Alternative polyadenylation is associated with lower expression of PABPN1 and poor prognosis in non-small cell lung cancer. Cancer Sci. 2014;105:1135-41.

13. Wu J, Jiang Z, Chen C, Hu Q, Fu Z, Chen J, et al. CircIRAK3 sponges miR-3607 to facilitate breast cancer metastasis. Cancer Lett. 2018:430:179-92.

14. Du WW, Yang W, Liu E, Yang Z, Dhaliwal P, Yang BB. Foxo3 circular RNA retards cell cycle progression via forming ternary complexes with p21 and CDK2. Nucleic Acids Res. 2016;44:2846-58.

15. Zhang M, Huang N, Yang X, Luo J, Yan S, Xiao F, et al. A novel protein encoded by the circular form of the SHPRH gene suppresses gliomatumorigenesis. Oncogene. 2018;37:1805-14.

16. Wang R, Zhang S, Chen X, Li N, Li J, Jia R, et al. CircNT5E acts as a sponge of miR-422a to promote glioblastoma tumorigenesis. Cancer Res. 2018;78:4812-25.

17. Bi J, Liu H, Dong W, Xie W, He Q, Cai Z, et al. Circular RNA circ-ZKSCAN1 inhibits bladder cancer progression through miR-1178-3p/p21 axis and acts as a prognostic factor of recurrence. Mol Cancer. 2019;18:133.
18. Chen Q, Liu T, Bao Y, Zhao T, Wang J, Wang H, et al. CircRNA cRAPGEF5 inhibits the growth and metastasis of renal cell carcinoma via the miR27a-3p/TXNIP pathway. Cancer Lett. 2020;469:68-77.

19. Huang W, Fang K, Chen TQ, Zeng ZC, Sun YM, Han C, et al. circRNA circAF4 functions as an oncogene to regulate MLL-AF4 fusion protein expression and inhibit MLL leukemia progression. J Hematol Oncol. 2019;12:103.

20. Jeck WR, Sorrentino JA, Wang K, Slevin MK, Burd CE, Liu J, et al. Circular RNAs are abundant, conserved, and associated with ALU repeats. RNA. 2013;19:141-57.

21. Zhang XO, Wang HB, Zhang Y, Lu X, Chen LL, Yang L. Complementary sequence-mediated exon circularization. Cell. 2014;159:134-47.

22. Liang D, Wilusz JE. Short intronic repeat sequences facilitate circular RNA production. Genes Dev. 2014;28:2233-47.

23. Conn SJ, Pillman KA, Toubia J, Conn VM, Salmanidis M, Phillips CA, et al. The RNA binding protein quaking regulates formation of circRNAs. Cell. 2015;160:1125-34.

24. circBase. http://www.circbase.org/cgi-bin/singlerecord.cgi?id=hsa_circ_ 0002346. Accessed. Accessed 27 Nov 2021.

25. Petar Glažar, Panagiotis Papavasileiou, Nikolaus Rajewsky. circBase: a database for circular RNAs. RNA. 2014;20:1666-70.

26. Prenkert M, Uggla B, Tidefelt U, Strid H. CRIM1 is expressed at higher levels in drug-resistant than in drug-sensitive myeloid leukemia HL60 cells. Anticancer Res. 2010;30:4157-61.

27. Lim B, Park JL, Kim HJ, Park YK, Kim JH, Sohn HA, et al. Integrative genomics analysis reveals the multilevel dysregulation and oncogenic characteristics of TEAD4 in gastric cancer. Carcinogenesis. 2014;35:1020-7.

28. Hudson BD, Hum NR, Thomas CB, Kohlgruber A, Sebastian A Collette NM, et al. SOST inhibits prostate Cancer invasion. PLoS One. 2015;10:e0142058.

29. Zeng H, Zhang Y, Yi Q, Wu Y, Wan R, Tang L. CRIM1, a newfound cancerrelated player, regulates the adhesion and migration of lung cancercells. Growth Factors. 2015;33:384-92

30. Circlnteractome. https://circinteractome.nia.nih.gov/. Accessed 21 June 2021.

31. Dudekula DB, Panda AC, Grammatikakis I, De S, Abdelmohsen K, Gorospe M. Circlnteractome: a web tool for exploring circular RNAs and their interacting proteins and microRNAs. RNA Biol. 2016;13:34-42.

32. TargetScanHuman. http://www.targetscan.org/vert_72/. Accessed 21 June 2021.

33. Agarwal V, Bell GW, Nam JW, Bartel DP. Predicting effective microRNA target sites in mammalian mRNAs. Elife. 2015;4:e05005.

34. Chen X, Dong C, Law PT, Chan MT, Su Z, Wang S, et al. MicroRNA-145 targets TRIM2 and exerts tumor-suppressing functions in epithelial ovarian cancer. Gynecol Oncol. 2015:139:513-9.

35. Hang W, Feng Y, Sang Z, Yang Y, Zhu Y, Huang Q, et al. Downregulation of miR-145-5p in cancer cells and their derived exosomes may contribute to the development of ovarian cancer by targeting CT. Int J Mol Med. 2019;43:256-66.

36. Xia X, Li X, Li F, Wu X, Zhang M, Zhou H, et al. A novel tumor suppressor protein encoded by circular AKT3 RNA inhibits glioblastoma tumorigenicity by competing with active phosphoinositide-dependent Kinase-1. Mol Cancer. 2019;18:131

37. Yang Y, Gao X, Zhang M, Yan S, Sun C, Xiao F, et al. Novel role of FBXW7 circular RNA in repressing glioma tumorigenesis. J Natl Cancer Inst. 2018;110:304-15.

38. CircRNA Db. http://reprod.njmu.edu.cn/cgi-bin/circrnadb/detail_info. php?circ_id=hsa_circ_16396. Accessed 27 Nov 2021.

39. Jiang J, Xie C, Liu Y, Shi Q, Chen Y. Up-regulation of miR-383-5p suppresses proliferation and enhances chemosensitivity in ovarian cancer cells by targeting TRIM27. Biomed Pharmacother. 2019;109:595-601.

40. Xu G, Li N, Zhang Y, Zhang J, Xu R, Wu Y. MicroRNA-383-5p inhibits the progression of gastric carcinoma via targeting HDAC9 expression. Braz Med Biol Res. 2019;52:e8341.

41. Wei C, Gao JJ. Downregulated miR-383-5p contributes to the proliferation and migration of gastric cancer cells and is associated with poor prognosis. PeerJ. 2019;7:e7882.

42. Prislei S, Martinelli E, Zannoni GF, Petrillo M, Filippetti F, Mariani M, et al. Role and prognostic significance of the epithelial-mesenchymal transition factor ZEB2 in ovarian cancer. Oncotarget. 2015;6:18966-79. 
43. Li Y, Fei H, Lin Q, Liang F, You Y, Li M, et al. ZEB2 facilitates peritoneal metastasis by regulating the invasiveness and tumorigenesis of cancer stem-like cells in high-grade serous ovarian cancers. Oncogene. 2021:40:5131-41.

44. Song Y, Jiang K, Su S, Wang B, Chen G. Clinical manifestations and epigenetic mechanisms of gastric mucosa associated lymphoid tissue lymphoma and long-term follow-up following helicobacter pylori eradication. Exp Ther Med. 2018;15:553-61.

\section{Publisher's Note}

Springer Nature remains neutral with regard to jurisdictional claims in published maps and institutional affiliations.

- fast, convenient online submission

- thorough peer review by experienced researchers in your field

- rapid publication on acceptance

- support for research data, including large and complex data types

- gold Open Access which fosters wider collaboration and increased citations

- maximum visibility for your research: over 100M website views per year

At BMC, research is always in progress.

Learn more biomedcentral.com/submissions 